\title{
Desempeño económico-financiero 2010-2019 de empresas longevas en el Ecuador: ¿Avance o retroceso?
}

\section{Economic-financial performance 2010-2019 of long-term companies in Ecuador: Advance or recession?}

Carla de los Ángeles Ojeda Zambrano ${ }^{1}$, Jairo Alexander Gutiérrez Burbano ${ }^{2}$, Luis Felipe Córdova Ballesteros ${ }^{3}$

INFORMACIÓN DEL

\section{ARTÍCULO}

Fecha de recepción: 7 de Septiembre de 2021.

Fecha de aceptación: 5 de Noviembre de 2021.

${ }^{1}$ Magíster en Gerencia de Negocios, UTE. Docente-investigador, Universidad Central del Ecuador - Ecuador.

E-mail: caojeda@uce.edu.ec

Código ORCID:

https://orcid.org/0000-0003-1435-2317

2 Magíster en Administración de Empresas, Universidad Central del Ecuador. Docente-investigador, Universidad Central del Ecuador - Ecuador

E-mail: jgutierrez@uce.edu.ec

Código ORCID:

https://orcid.org/0000-0001-5655-4588

${ }^{3}$ Master Real Estate Management and Project Development, Universidad San Francisco de Quito. Docente-investigador, Universidad Central del Ecuador Ecuador.

E-mail: lfcordova@uce.edu.ec Código ORCID:

https://orcid.org/0000-0003-0573-3572

CITACIÓN: Ojeda, C., Gutiérrez, J., \& Córdova, L. (2021). Desempeño económico-financiero 2010-2019 de empresas longevas en el Ecuador: ¿Avance o retroceso?. Podium, 40, 89-104.

doi:10.31095/podium.2021.40.6

\section{ENLACE DOI:}

http://dx.doi.org/10.31095/podium.202 1.40 .6

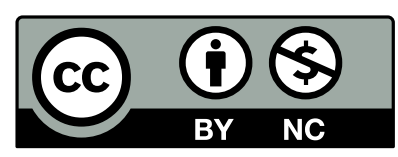

\section{Resumen}

El presente estudio analiza el desempeño económico-financiero en el período 2010-2019 de un conjunto de 155 empresas ecuatorianas longevas con más de 50 años de trayectoria, el mismo que se desarrolla a través de una metodología de tipo descriptiva longitudinal y enfoque inductivo con base cuantitativa. El grupo estudiado muestra un incremento de su activo y patrimonio; sin embargo, registra grandes fluctuaciones en sus utilidades que provocan que tanto el ROA como el ROE presenten una tendencia decreciente en determinados años, por lo cual se concluye que, de acuerdo al tamaño, las organizaciones más afectadas son las microempresas y en relación al tipo, las más frágiles son las empresas de servicios.

\section{Palabras Clave:}

Perdurabilidad empresarial, activo, patrimonio, beneficios, rentabilidad, finanzas empresariales.

\section{Clasificación JEL: M21.}

\begin{abstract}
This study analyzes the economic-financial performance in the period 2010-2019 of a group of 155 long-standing Ecuadorian companies with more than 50 years of experience, which is developed through a longitudinal descriptive methodology and an inductive approach based on quantitative. The group studied shows an increase in its assets and equity; however, it registers large fluctuations in its profits that cause both the ROA and the ROE to present a decreasing trend in certain years, for which it is concluded that, according to size, the most affected organizations are micro-enterprises and in relation to the type, the most fragile are the service companies.
\end{abstract}

\section{Keywords:}

Business perdurability, assets, equity, profits, profitability, business finances.

JEL Classification: M21. 


\section{Introducción}

Desde el punto de vista administrativo una empresa es una entidad creada para un fin específico, por lo general para el logro de algún tipo de rentabilidad mientras que desde el punto de vista orgánico, se constituye en un sistema como tal, entendiéndolo como un conjunto interrelacionado de partes de menor tamaño que cumplen un objetivo particular y que a su vez forma parte de un sistema mayor o macrosistema, "por eso es relevante hablar de capacidad de conexión o de enlace, porque las estructuras se determinan por su capacidad para establecer nexos. Ya no se refieren a invariantes, sino a operaciones y a enlaces" (Varela, 1995, pág. 5).

En el Ecuador existen diversos tipos de empresas, las cuales se clasifican en diferentes tamaños desde las micro, pequeñas, medianas hasta grandes empresas según el Reglamento de inversiones del Código Orgánico de la Producción, Comercio e Inversiones, COPCI (Asamblea Nacional, 2018, pág. 40). El mencionado Reglamento indica que "en caso de inconformidad frente a las variables aplicadas, se estará a lo señalado en el inciso segundo del Artículo 53 del Código de la Producción, Comercio e Inversiones". Efectivamente, el Artículo. 53, Definición y clasificación de las MIPYMES del COPCI (Asamblea Nacional, 2019, pág. 26) indica que "en caso de inconformidad de las variables aplicadas, el valor bruto de las ventas anuales prevalecerá sobre el número de trabajadores, para efectos de determinar la categoría de una empresa".
El presente estudio considera a todos los tamaños de empresas provenientes de los diferentes tipos y sectores productivos; sin embargo, el interés principal tiene que ver con aquellas empresas que han superado al menos cincuenta años de vida institucional, considerando su fortaleza para afrontar los retos de permanencia en el mercado, lo cual se ha estudiado en la literatura incluso a nivel latinoamericano lo que significa que mientras mayor sea el tamaño de la organización, mayor será la probabilidad de supervivencia. "El modelo de aprendizaje pasivo de Jovanovic (1982) llega a la conclusión de que, en la medida en que una firma opera durante más tiempo en el mercado, más experiencia adquiere y más eficiente se vuelve... a mayor tamaño, menos probabilidades de cierre" (Parra, 2011, pág. 30).

El objetivo de este estudio descriptivo transversal es medir y describir el desempeño económico-financiero de las empresas longevas de la provincia de Pichincha, Ecuador, durante los años 2010-2019 y determinar si existe un avance o retroceso en sus principales variables internas. El estudio se focaliza en una de las provincias del país que concentra una gran cantidad de empresas de diverso tamaño (micro, pequeñas, medianas y grandes) y tipo (industriales, comerciales y de servicios). En la provincia de Pichincha, en donde se encuentra la capital del país, Quito, funcionan empresas de diversos sectores de la Clasificación Industrial Internacional Uniforme (CIIU) utilizada por la Superintendencia de Compañías, Valores 
y Seguros (Camino, Reyes, Apraes, Bravo, y Herrera, 2017) tales como agricultura, ganadería, industrias manufactureras, construcción, comercio al por mayor y menor, turismo, transporte, comunicación, financieras, inmobiliarias, enseñanza, salud, artes, entre otras.

\section{Revisión de literatura}

Existen muchos factores diferentes al tamaño que influyen en la permanencia de las empresas y por ende en su longevidad; sin embargo, su dimensión les otorga mayor capacidad de conocer sus fortalezas y debilidades, y con ello determinar mejores estrategias para solventar las dificultades del entorno y evitar su cierre. En el Ecuador no se evidencian estudios amplios sobre la longevidad de sus empresas sino sobre todo análisis de conglomerados empresariales de diverso tipo y edad, tanto generales como específicos, inclusive provenientes de diferentes localizaciones geográficas como se expone más adelante, razón por la cual el presente estudio se circunscribe a las empresas de mayor antigüedad, es decir aquellas que tienen al menos cincuenta años de funcionamiento al constituirse jurídicamente antes de 1970. El interés de estudiar las empresas longevas se debe a que ha sido notable su capacidad para afrontar a lo largo de cincuenta años hasta la actualidad los diferentes retos del entorno socioeconómico del país como los que se reseñan a continuación.

El Ecuador de los últimos cincuenta años ha vivido una serie de eventos socioeconómicos y políticos de gran importancia, siendo los más relevantes el retorno a la democracia a finales de los años 70 y la crisis bancaria de finales de los años 90, los cuales implicaron gran conmoción en el tejido empresarial del país, ocasionado incluso que varias organizaciones ya creadas se extingan por su debilidad o incapacidad para enfrentar tales cambios. Ello significa que las empresas longevas en el Ecuador han afrontado durante muchas décadas los principales hitos de la historia contemporánea nacional desde la dictadura militar (Arregui, 1999) y el proceso de reestructuración jurídica del Estado (Sierra, 1999); el juego del recambio 'democrático' entre sectores de izquierda y derecha (Ayala, 1993) hasta el final del Siglo XX en donde la presidencia de Jamil Mahuad desencadenó "la medida monetaria más radical para la nación al decretar la dolarización de la economía, convirtiéndose el Ecuador en el primer país latinoamericano que elimina su moneda nacional” (Larrea, 2004, págs. 43-53).

Ya en el Siglo XXI y con un país dolarizado vendrían gobiernos mayormente inconclusos por problemas socioeconómicos y políticos hasta el ingreso al poder de Rafael Correa Delgado quien gobernó consecutivamente desde el 15 de enero de 2007 al 24 de mayo de 2017, con un régimen proveniente del socialismo del siglo XXI en el cual, si bien se ejecutaron obras de infraestructura, prevaleció el incremento del tamaño del estado y la concentración de poder que se logró fraguar progresivamente en un amplio período de diez años. Según Fierro (2019a, pág. 53) 
"En la década del Gobierno de Rafael Correa, los principales grupos económicos duplicaron sus ingresos. Entre 2013 y 2017, los ingresos de los 27 principales grupos económicos aumentaron de USD 25.686 millones a USD 28.689 millones. Su participación en el VAB del país aumentó del $28,4 \%$ en 2013 a 29,6\% en 2017". Posteriormente asume el gobierno de Lenin Moreno Garcés desde el 24 de mayo de 2017 hasta culminar su período en 2021, quien enfrentó un gran déficit fiscal, levantamientos populares y la pandemia mundial Covid 19.

Al transcurrir los mencionados hechos históricos y en particular 20 años de la dolarización, existen muchos criterios a favor y en contra. Algunos parámetros influyeron en el desarrollo socioeconómico. Considerando la inflación acumulada desde el año 1980 hasta el año 2019, en el año 2000 la inflación llegó al 91\% para luego bajar drásticamente al $22 \%$ y continuar en una franja menor al $10 \%$, incluso con valores negativos del $-0,20$ en el año 2017 al finalizar su periodo Rafael Correa y del $-0,07$ en el 2019 , lo cual evidencia el efecto estabilizador de la dolarización (INEC, 2020). Respecto al Producto Interno Bruto (PIB), la tasa de variación para el período comprendido entre 1999 a 2018 demuestra que en 1999 se registró un decrecimiento de $3,6 \%$, pasando a las cotas máximas del $8 \%$ y $7,8 \%$ en el 2004 y 2011, respectivamente. El comportamiento del PIB ha sido sumamente volátil; factores externos como internos han impactado directamente en su comportamiento, por lo que muestran una economía que aún está lejos del crecimiento sostenido (BCE, 2020).

En base a lo referido por Acosta-González y Camelos (2018, pág. 17) "el Estado se encuentra en un contexto en el que no tiene otra alternativa que no sea reducir gradualmente su participación y presionar a la transición hacia una economía liderada por el sector privado". Ante este panorama, Campuzano, Cedillo, Chuquirima, y Romero (2019, pág. 52) afirman que ante la "grave y larga crisis económica, con agudas consecuencias sociales, por la que atraviesa nuestro país desde el año 2008, la recién aprobada Ley 14/2013, de 27 de septiembre, de apoyo a los emprendedores y su internacionalización, pretende invertir esta situación". Otro aporte relevante en materia de intervención gubernamental según Fierro (2019b, pág. $173)$ es la constitución de "la Superintendencia de Control de Poder de Mercado, junto a la aprobación de su respectiva Ley, la cual ha impulsado algunas políticas anti-monopólicas y pro-competitivas". Por su parte, el nuevo Código de Comercio "a pesar de que alude a la libertad de asociación empresarial, mantiene una fuerte tendencia regulatoria y tiende a la armonización con el régimen tributario, así como el de control de poder de mercado" (Lascano, Méndez, y Barcia, 2020, pág. 74), lo cual trae varios beneficios para el ecosistema empresarial del Ecuador. Es interesante contrastar que, a pesar de las crisis, muchas empresas también han crecido y según 
Mejía, Pinos, Proaño, y Rojas (2019, pág. 13) existe relación directa entre la confianza y el desarrollo, es decir "para la economía ecuatoriana en el periodo 2007-2018, existe una relación causal entre la confianza empresarial, y el ciclo económico, así por cada punto porcentual que aumente la confianza empresarial del trimestre anterior la producción en ese periodo aumenta en $0,17 \%$ ".

Respecto al emprendimiento $\mathrm{y}$ crecimiento económico, en regiones como la litoral, específicamente en la provincia del Guayas, se evidencia que "el emprendimiento temprano y las empresas establecidas impulsan el crecimiento económico de la provincia como un todo armónico que propicia lo nuevo y mantiene lo existente, de modo que convierte a esta provincia en un polo de desarrollo económico del Ecuador" (Moina-Sánchez, Morales-Carrasco, y Córdova-Pacheco, 2020, pág. 79). Por su parte, en la región interandina, específicamente en la Sierra central, cantón Riobamba, el crecimiento se focalizó principalmente en actividades de construcción, industriales y de transporte en las cuales las "políticas implementadas durante el 'Correato' provocaron un aumento de la deuda pública para sostener el crecimiento económico. No obstante, a partir de 2015, el crecimiento se detiene, y las variables económicas y sociales empiezan a retroceder" (Vives y Naranjo, 2020, pág. 69). En el estudio de Chuncho, Uriguen, y Apolo (2021, pág. 16) sobre el desarrollo agropecuario e industrial ecuatoriano en el período 2000-2018 se afirma que en base a una comparación de ambos sectores respecto a cuál ha contribuido en mayor medida al PIB, "ha sido el sector industrial... su aporte promedio ha sido de \$27.634.967 millones en relación al sector agropecuario de \$10.199.659 millones con una diferencia en estos años de \$17.435.308 millones".

En relación a la rentabilidad, en especial de las empresas industriales, en el estudio de Coello (2017, pág. 69) se concluye que "la relación encontrada entre la estructura del mercado y el desempeño de las empresas es positiva y estadísticamente significativa en el conjunto de la industria manufacturera, sin embargo, el efecto difiere entre regiones, tipo de empresas y sectores". Respecto a las empresas pequeñas, el mismo análisis indica que "a pesar de que la estructura de mercado tiene una relación positiva con el desempeño de las empresas pequeñas, el hacedor de política pública debe formular las condiciones e incentivos necesarios para minimizar el riesgo de cierre de estos emprendimientos" con lo cual concluye que "las empresas pequeñas son las más sensibles ante el ingreso de nuevos competidores, lo que implica que la protección desde la política pública... debe ayudar a enfrentar las barreras de entrada al mercado". En la misma línea de las empresas ecuatorianas de tamaño reducido, Solís y Robalino (2019, págs. $85,91)$ sostienen que han tenido que afrontar "un escenario complicado tanto por la situación económica como por la falta de políticas de incentivos a la producción que registraron. El dinamismo de una economía depende de la capacidad de promover, sustentar y reconocer el 
crecimiento de los negocios". Las principales causas de su bajo desempeño son: "(1) retraso tecnológico (2) falta de asociatividad de las PYMES evidenciando una deficiencia en la gestión interna y dificultad para enfrentar las competencias nacionales e internacionales". En relación a la rentabilidad en el período 2016-2017 de las empresas de construcción ecuatorianas, según Arévalo, Osejo, Fonseca, y Canchignia (2018, págs. 47-48) "en el año 2016 en lo que se refiere a la Rentabilidad sobre el Patrimonio (ROE), éste tuvo un resultado de $-45 \%$ mientras que en lo que respecta a la Rentabilidad sobre el Activo (ROA), el resultado fue de $-18 \%$ con respecto al activo total". En el año 2017 en términos de ROE "los socios o dueños de los negocios obtuvieron un rendimiento sobre la inversión de $15 \%$ " y en relación al ROA, la cifra "corresponde al $6 \%$ con respecto al total activo", es decir una mejoría sobre el año anterior.

Otro aspecto de gran importancia para comprender el panorama socioeconómico que las empresas longevas han enfrentado es la inversión externa como uno de los factores dinamizadores de la economía de un país, más aún cuando la moneda es fuerte como el dólar. El Ecuador actualmente es uno de los destinos preferidos en América Latina y el Caribe para los inversionistas extranjeros, además en este último año se ha incrementado notablemente, tal como lo presenta en su informe anual de 2019 la Comisión Económica para América Latina y el Caribe -CEPAL (2019).

Según informe del INEC (2017, pág.
99) el comportamiento y evolución del número de empresas desde el 2012 hasta el 2018 tiene una creciente y constante tendencia en la creación de nuevos negocios y de hecho en el Ecuador el nivel de emprendimiento es uno de los más altos en Latinoamérica, lo cual dinamiza las relaciones con las organizaciones longevas. Con esta orientación se puede pronosticar que en el futuro se incrementará el número de empresas siempre que los factores productivos permanezcan ciertamente estables. Una inflación relativamente baja ha permitido conservar el poder monetario; sin embargo, los problemas de desempleo y subempleo no han podido ser superados, más aún con la migración de las últimas décadas.

En los próximos años se prevén incrementos del número de empresas; sin embargo, el $90,81 \%$ se refiere a microempresas lo cual implica cierta preocupación pues de acuerdo al INEC (2017, pág. 99), "la tasa de riesgo de las empresas disminuye a una tasa decreciente con el crecimiento del sector; la tasa de riesgo es menor para empresas en sectores grandes; las unidades económicas más formales (sociedades o personas que llevan contabilidad) tienen menor riesgo de desaparecer", por lo que las empresas de menor tamaño se muestran más vulnerables y por ende el panorama empresarial y laboral se vuelve incierto, a la espera de políticas públicas estables y de emprendimientos sólidos.

En estos escenarios y en el pasado reciente, una gran cantidad de empresas ecuatorianas de diverso tamaño, tipo de 
actividad y características no lograron blindar su estructura contra estos factores exógenos y se vieron afectadas hasta el punto de la quiebra y cierre societario, cuyos efectos en la economía, componente laboral $\mathrm{y}$ tejido social dejaron secuelas graves, lo cual demuestra que el tamaño o dimensión no es el único factor que puede incidir en su supervivencia. Solamente las empresas que lograron formar con mayor solidez determinados factores de competitividad pudieron enfrentar las mencionadas crisis $\mathrm{y}$, sin dejar por supuesto de verse afectadas, continuaron aportando positivamente a la economía del país especialmente durante los últimos diez años que constituye el período de análisis de este estudio. De hecho, la longevidad empresarial es una característica en realidad difícil de conseguir pues lo impiden muchos factores dando como resultado que una proporción muy pequeña del conglomerado empresarial supere el medio siglo de vida. En forma comparativa en el mundo, por ejemplo, España, (Aguiar, 2018, págs. 138-139) enfatiza que "Fariñas y Huergo (2015) concluyen que en España existe una gran turbulencia empresarial, cada año más del $20 \%$ de las empresas existentes a comienzos de año son dadas de alta o baja", aunque incluso tengan un nivel de emprendimiento joven mayor que países vecinos. Por otro lado, agrega que "según el informe elaborado por Crédito y Caución (2014), la mortalidad de empresas es elevada... A finales de 2016, solo un $16,8 \%$ de las empresas españolas tienen 20 o más años". Esta realidad tiene muchas similitudes con respecto a contextos más cercanos como el de
México pues "el promedio de esperanza de vida de una empresa en México es de 7,8 años, a los 5 años de su creación desaparece el $65 \%$ y a los 25 años cierran o quiebran el $89 \%$ de los negocios", de acuerdo con lo mencionado por Rodríguez y García (2020, pág. 6) según los datos del INEGI (Instituto Nacional de Estadística y Geografía). Tal como se puede observar, la baja proporción de organizaciones longevas es similar en otras latitudes, lo cual abona al análisis de las empresas del presente estudio.

\section{Metodología}

El estudio es de tipo descriptivo y longitudinal, ya que analiza el desempeño económico-financiero de las empresas longevas a través de los últimos diez años consecutivos de operaciones. Es una investigación documental debido a que la recopilación se realizó con base en datos secundarios de la Superintendencia de Compañías, Valores y Seguros del Ecuador. Dentro de la trayectoria de investigación, el método utilizado es inductivo con base cuantitativa debido a la naturaleza de la información necesaria para la consecución del objetivo, lo cual implica medir el nivel desempeño económico- financiero de las empresas tanto por su tamaño como por su tipo de actividad en lo que respecta a Activo, Pasivo, Patrimonio, Ingresos, Utilidades/ Pérdidas, ROA y ROE.

La hipótesis de tipo descriptiva del estudio es la siguiente: Las empresas mayores a 50 años de diverso tamaño y tipo de la Provincia de Pichincha que lograron permanecer en el mercado hasta 
la actualidad registran un crecimiento sostenido de su activo, patrimonio, utilidades, y por ende de su ROA y ROE.

En base a esta hipótesis se analiza el desempeño del conjunto de empresas en forma general y también de forma particularizada, segmentando por tamaño y tipo de organizaciones para profundizar el estudio. De acuerdo al tipo de investigación, no aplica la selección de muestra pues se trabajó con el total de empresas que cumplían con la característica de longevidad, es decir encontrarse vigentes hace más de cincuenta años $y$ haber reportado información completa a la Superintendencia de Compañías, Valores y Seguros. En tal razón, el total de empresas que cumple ambos factores corresponde a 155 , cuya información fue trasladada a la base de datos de la investigación. Los datos fueron procesados en el paquete Estadístico y su complemento gráfico en el programa Excel. La información de las diferentes empresas es de carácter informativo, legal y económico, a partir de la cual se calcularon los respectivos promedios por tamaño y tipo de organizaciones.

Tabla 1.

Activo promedio por tamaño de empresa (US\$)

\begin{tabular}{cccccc}
\hline Año & Micro & Pequeña & Mediana & Grande & Total general \\
\hline 2010 & $185.195,84$ & $504.922,66$ & $1.744 .108,37$ & $41.445 .123,02$ & $20.007 .713,99$ \\
\hline 2011 & $201.600,05$ & $565.738,23$ & $1.832 .758,79$ & $49.855 .580,53$ & $24.003 .750,24$ \\
\hline 2012 & $309.676,23$ & $1.005 .221,34$ & $2.750 .741,78$ & $53.556 .831,14$ & $26.052 .790,52$ \\
\hline 2013 & $318.808,83$ & $1.029 .487,57$ & $3.018 .213,18$ & $59.118 .867,47$ & $28.731 .361,07$ \\
\hline 2014 & $317.707,29$ & $1.327 .060,51$ & $2.983 .670,72$ & $64.649 .192,66$ & $31.411 .768,73$ \\
\hline 2015 & $263.946,93$ & $1.276 .517,29$ & $3.130 .043,08$ & $66.747 .027,97$ & $32.410 .968,39$ \\
\hline 2016 & $311.383,46$ & $1.234 .717,68$ & $3.141 .693,70$ & $69.566 .475,77$ & $33.732 .250,67$ \\
\hline 2017 & $332.729,33$ & $1.240 .688,46$ & $3.059 .593,10$ & $73.143 .858,64$ & $35.404 .088,01$ \\
\hline 2018 & $324.499,90$ & $1.269 .126,33$ & $3.232 .932,37$ & $77.394 .256,58$ & $37.446 .849,24$ \\
\hline 2019 & $298.737,32$ & $1.272 .547,52$ & $3.088 .801,70$ & $83.085 .018,55$ & $40.098 .571,51$ \\
\hline Promedio & $286.428,52$ & $1.072 .602,76$ & $2.798 .255,68$ & $63.856 .223,23$ & $30.930 .011,24$ \\
\hline
\end{tabular}

Fuente: Superintendencia de Compañías, Valores y Seguros (2020).

\section{6}

PODIUM No. 40, Diciembre 2021, pp. 89-104

(C) Universidad Espíritu Santo - UEES

ISSN: 1390-5473 e-ISSN: 2588-0969

\section{Resultados}

Las empresas analizadas se encuentran en los cantones Quito y Rumiñahui de la provincia de Pichincha. Del conjunto de 155 empresas, $34,19 \%$ se crearon en el periodo 1921-1960, mientras que $65,81 \%$ se crearon en la década 1961-1970. Respecto a la constitución legal, el $79,35 \%$ están constituidas como Sociedades Anónimas, y solamente el 35,48\% tienen inversión extranjera. En relación al tamaño: 5,81\% son microempresas; $27,74 \%$ son empresas pequeñas; $19,35 \%$ son empresas medianas $\mathrm{y}, 47,10 \%$ son empresas grandes. Respecto al sector, $46,45 \%$ corresponde a empresas industriales; $32,90 \%$, a empresas comerciales y el $20,65 \%$, son empresas de servicios. Por último, en relación al número de empleados, el 29,68\% presentan una nómina con un máximo de 9 empleados; 48,39\% tienen entre 10 y 199 trabajadores, y 21,93\% cuentan con 200 o más colaboradores.

Crecimiento empresarial considerando el activo

Según la Tabla 1 en los cuatro 
tamaños de empresas micro, pequeñas, medianas y grandes el crecimiento del activo es sostenido sin fluctuaciones ni oscilaciones marcadas.

Se puede observar en la Tabla 2 que, en los tres tipos de empresas industriales, comerciales y de servicios el crecimiento del activo es constante y no se registran fluctuaciones ni oscilaciones.

\section{Crecimiento empresarial considerando} el patrimonio

En las empresas grandes el patrimonio Tabla 2 .

Activo promedio por tipo de empresa (US\$)

\begin{tabular}{ccccc}
\hline Año & Industrial & Comercial & Servicios & Total general \\
\hline 2010 & $19.416 .197,19$ & $30.328 .795,59$ & $4.889 .403,00$ & $20.007 .713,99$ \\
\hline 2011 & $24.441 .742,56$ & $35.075 .836,44$ & $5.372 .130,13$ & $24.003 .750,24$ \\
\hline 2012 & $26.099 .436,86$ & $38.202 .004,29$ & $6.585 .026,79$ & $26.052 .790,52$ \\
\hline 2013 & $29.705 .760,85$ & $41.051 .735,75$ & $6.903 .364,42$ & $28.731 .361,07$ \\
\hline 2014 & $32.159 .456,28$ & $45.404 .113,44$ & $7.429 .172,36$ & $31.411 .768,73$ \\
\hline 2015 & $33.338 .008,33$ & $47.101 .637,33$ & $6.911 .874,91$ & $32.410 .968,39$ \\
\hline 2016 & $34.344 .545,59$ & $49.371 .239,26$ & $7.429 .949,01$ & $33.732 .250,67$ \\
\hline 2017 & $35.556 .705,94$ & $52.797 .553,89$ & $7.339 .861,39$ & $35.404 .088,01$ \\
\hline 2018 & $36.781 .151,52$ & $56.953 .785,71$ & $7.855 .489,12$ & $37.446 .849,24$ \\
\hline 2019 & $36.233 .996,60$ & $65.900 .430,86$ & $7.672 .151,73$ & $40.098 .571,51$ \\
\hline Promedio & $30.807 .700,17$ & $46.218 .713,26$ & $6.838 .842,29$ & $30.930 .011,24$ \\
\hline
\end{tabular}

Fuente: Superintendencia de Compañías, Valores y Seguros (2020).

Tabla 3.

Patrimonio promedio por tamaño de empresa (US\$)

\begin{tabular}{cccccc}
\hline Año & Micro & Pequeña & Mediana & Grande & Total general \\
\hline 2010 & $139.245,89$ & $280.517,07$ & $884.672,22$ & $21.305 .451,99$ & $10.291 .313,60$ \\
\hline 2011 & $144.437,40$ & $306.601,02$ & $908.803,61$ & $25.744 .088,80$ & $12.393 .976,59$ \\
\hline 2012 & $252.191,50$ & $731.518,81$ & $1.586 .743,78$ & $28.457 .925,58$ & $13.927 .457,50$ \\
\hline 2013 & $258.771,54$ & $760.710,59$ & $1.669 .419,32$ & $32.990 .252,13$ & $16.086 .519,25$ \\
\hline 2014 & $257.838,90$ & $998.914,00$ & $1.633 .088,90$ & $36.255 .887,44$ & $17.683 .524,53$ \\
\hline 2015 & $202.128,44$ & $970.509,71$ & $1.710 .233,79$ & $38.337 .291,22$ & $18.667 .615,14$ \\
\hline 2016 & $245.410,30$ & $951.591,04$ & $1.581 .813,87$ & $40.151 .816,63$ & $19.494 .607,34$ \\
\hline 2017 & $264.734,09$ & $918.455,02$ & $1.620 .210,99$ & $42.935 .171,18$ & $20.804 .838,70$ \\
\hline 2018 & $261.167,47$ & $1.004 .306,18$ & $1.708 .463,04$ & $45.442 .684,48$ & $22.026 .487,30$ \\
\hline 2019 & $224.836,00$ & $1.012 .260,30$ & $1.697 .929,27$ & $47.526 .588,31$ & $23.005 .997,05$ \\
\hline Promedio & $225.076,15$ & $793.538,37$ & $1.500 .137,88$ & $35.914 .715,78$ & $17.438 .233,70$ \\
\hline
\end{tabular}

Fuente: Superintendencia de Compañías, Valores y Seguros (2020).

promedio tiene una tendencia de crecimiento sostenido sin fluctuaciones ni oscilaciones según se observa en la Tabla 3. Por su parte, en las empresas micro, pequeñas $y$ medianas el patrimonio promedio tiene una tendencia de crecimiento con fluctuaciones $u$ oscilaciones por encima y por debajo de la recta de tendencia, es decir, su comportamiento es más irregular. El total general muestra una tendencia creciente.

En base a lo indicado en la Tabla 4, en las empresas industriales y comerciales el patrimonio promedio tiene una tendencia 
de crecimiento sostenido sin fluctuaciones ni oscilaciones. Por su parte, las empresas de servicios, si bien tienen una tendencia de crecimiento, también presentan fluctuaciones u oscilaciones, especialmente entre los años 2015 a 2018. El total general muestra una tendencia creciente, la cual corresponde al análisis de patrimonio promedio de la sección anterior.

\section{Crecimiento empresarial considerando} la utilidad

Respecto a las utilidades promedio, los cuatro grupos de empresas micro, pequeñas, medianas y grandes tienen una tendencia de leve crecimiento, pero con muy marcadas fluctuaciones $\mathrm{u}$ oscilaciones especialmente en el año 2012 y en el período entre el 2015 y el 2019, según se desprende de la Tabla 5. El total general muestra una dirección creciente; sin embargo, su línea de tendencia marca una ralentización en el nivel de resultados, los cuales incluyen no únicamente los de tipo positivo (beneficios) sino también los de tipo negativo (pérdidas). Las empresas más afectadas son las micro y pequeñas empresas, realidad muy similar a lo Tabla 4.

Patrimonio promedio por tipo de empresa (US\$)

\begin{tabular}{ccccc}
\hline Año & Industrial & Comercial & Servicios & Total general \\
\hline 2010 & $7.943 .677,35$ & $18.361 .992,10$ & $2.710 .851,32$ & $10.291 .313,60$ \\
\hline 2011 & $10.167 .290,59$ & $21.418 .098,41$ & $3.021 .825,94$ & $12.393 .976,59$ \\
\hline 2012 & $11.546 .745,95$ & $23.764 .040,31$ & $3.607 .004,66$ & $13.927 .457,50$ \\
\hline 2013 & $13.888 .023,98$ & $26.870 .645,98$ & $3.845 .931,64$ & $16.086 .519,25$ \\
\hline 2014 & $15.171 .130,95$ & $29.853 .811,82$ & $3.940 .014,70$ & $17.683 .524,53$ \\
\hline 2015 & $16.287 .914,67$ & $31.386 .029,31$ & $3.751 .968,61$ & $18.667 .615,14$ \\
\hline 2016 & $16.941 .295,63$ & $33.175 .288,67$ & $3.435 .972,81$ & $19.494 .607,34$ \\
\hline 2017 & $17.892 .958,39$ & $36.036 .540,35$ & $3.081 .044,89$ & $20.804 .838,70$ \\
\hline 2018 & $18.534 .525,83$ & $38.467 .748,63$ & $3.680 .140,37$ & $22.026 .487,30$ \\
\hline 2019 & $19.041 .306,15$ & $40.739 .988,07$ & $3.663 .003,35$ & $23.005 .997,05$ \\
\hline Promedio & $14.741 .486,95$ & $30.007 .418,37$ & $3.473 .775,83$ & $17.438 .233,70$ \\
\hline
\end{tabular}

Fuente: Superintendencia de Compañías, Valores y Seguros (2020).

Tabla 5.

Utilidad promedio por tamaño de empresa (US\$)

\begin{tabular}{rrrrrr}
\hline Año & Micro & Pequeña & Mediana & Grande & Total general \\
\hline 2010 & $9.099,79$ & $40.024,94$ & $145.612,69$ & $3.349 .129,11$ & $1.617 .146,94$ \\
\hline 2011 & $11.808,57$ & $51.667,28$ & $108.634,59$ & $6.225 .789,63$ & $2.968 .191,30$ \\
\hline 2012 & $15.781,34$ & $43.115,68$ & $99.306,31$ & $4.006 .954,75$ & $1.919 .244,47$ \\
\hline 2013 & $9.642,98$ & $40.530,72$ & $106.497,82$ & $5.031 .049,66$ & $2.401 .878,50$ \\
\hline 2014 & $5.934,99$ & $31.666,51$ & $132.514,02$ & $6.467 .611,00$ & $3.080 .813,54$ \\
\hline 2015 & $6.601,32$ & $33.014,19$ & $106.344,66$ & $5.978 .727,46$ & $2.845 .912,69$ \\
\hline 2016 & $5.225,68$ & $28.117,00$ & $29.289,61$ & $5.040 .084,86$ & $2.387 .489,97$ \\
\hline 2017 & $8.387,22$ & $49.281,18$ & $105.465,29$ & $5.390 .262,91$ & $2.573 .211,14$ \\
\hline 2018 & $2.427,81$ & $27.712,23$ & $112.589,24$ & $6.183 .398,17$ & $2.941 .801,42$ \\
\hline 2019 & 897,79 & $30.745,95$ & $109.470,54$ & $4.998 .800,13$ & $2.384 .043,11$ \\
\hline Promedio & $7.580,75$ & $37.587,57$ & $105.572,48$ & $5.267 .180,77$ & $2.511 .973,31$ \\
\hline
\end{tabular}

Fuente: Superintendencia de Compañías, Valores y Seguros (2020). 
observado por Solis y Robalino (2019), mientras que las más fuertes son las empresas grandes, lo cual coincide con lo encontrado por Parra (2011) y Mejía et al. (2019).

Al analizar las empresas por su tipo, en la Tabla 6 se observa que en los tres tipos: industrial, comercial y de servicios las utilidades promedio tienen una tendencia de crecimiento, pero con importantes fluctuaciones $\mathrm{u}$ oscilaciones especialmente en el año 2012 y el período comprendido entre el 2014 y el 2019, lo

Tabla 6.

Utilidad promedio por tipo de empresa (US\$)

\begin{tabular}{ccccc}
\hline Año & Industrial & Comercial & Servicios & Total general \\
\hline 2010 & $886.059,16$ & $3.469 .988,78$ & $309.127,75$ & $1.617 .146,94$ \\
\hline 2011 & $1.377 .493,84$ & $6.951 .673,16$ & $198.586,35$ & $2.968 .191,30$ \\
\hline 2012 & $1.439 .643,29$ & $3.517 .726,20$ & $450.766,85$ & $1.919 .244,47$ \\
\hline 2013 & $2.174 .457,64$ & $4.004 .474,52$ & $359.438,04$ & $2.401 .878,50$ \\
\hline 2014 & $2.969 .864,19$ & $4.850 .747,93$ & $509.616,64$ & $3.080 .813,54$ \\
\hline 2015 & $2.661 .415,00$ & $4.680 .840,47$ & $336.616,34$ & $2.845 .912,69$ \\
\hline 2016 & $2.314 .780,37$ & $3.978 .650,23$ & $15.174,89$ & $2.387 .489,97$ \\
\hline 2017 & $2.369 .723,64$ & $4.578 .689,93$ & $-165.173,81$ & $2.573 .211,14$ \\
\hline 2018 & $2.834 .356,15$ & $4.791 .608,05$ & $235.423,96$ & $2.941 .801,42$ \\
\hline 2019 & $1.847 .964,16$ & $4.439 .285,72$ & $314.677,83$ & $2.384 .043,11$ \\
\hline Promedio & $2.087 .575,74$ & $4.526 .368,50$ & $256.425,48$ & $2.511 .973,31$ \\
\hline
\end{tabular}

Fuente: Superintendencia de Compañías, Valores y Seguros (2020).

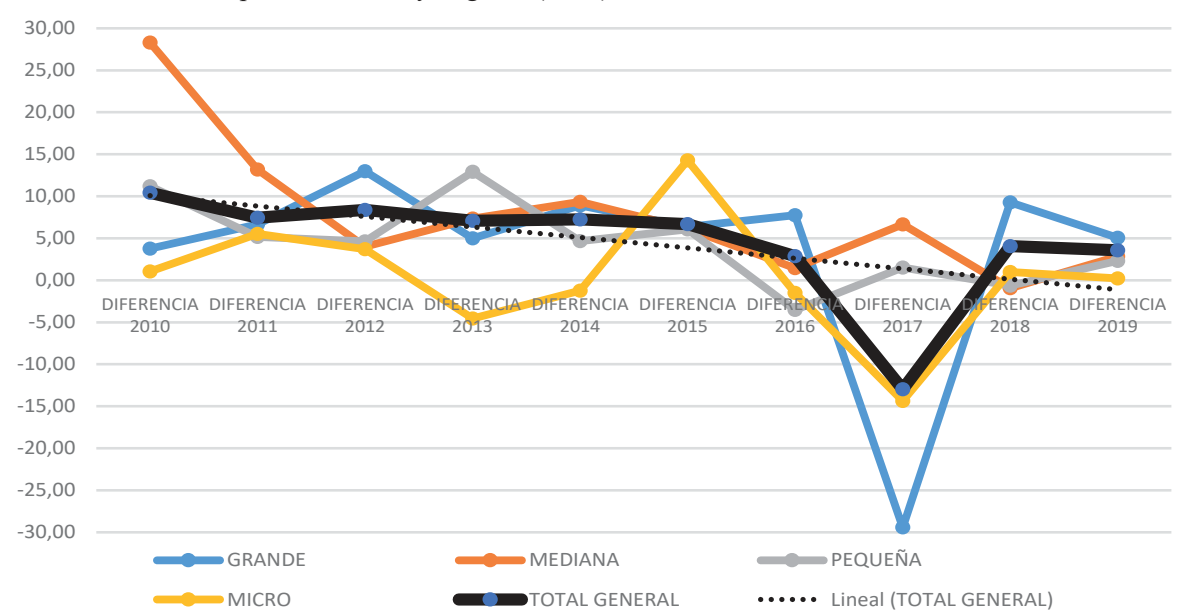

Figura 1. Diferencia ROE y ROA - período 2010-2019 - total empresas por tamaño.

Fuente: Superintendencia de Compañías, Valores y Seguros (2020).

99

PODIUM No. 40, Diciembre 2021, pp. 89-104

(C) Universidad Espíritu Santo - UEES

ISSN: 1390-5473 e-ISSN: 2588-0969 cual implica no solo la existencia de beneficios sino también considerables pérdidas. El total general muestra una tendencia creciente, aunque no muy marcada, la cual corresponde al análisis de utilidades promedio de la sección anterior. Las empresas comerciales que agrupan grandes conglomerados corporativos tienen un desempeño importante, lo cual coincide con lo señalado por Fierro (2019a).

\section{Análisis de rentabilidad ROA y $R O E$}

De acuerdo a la Figura 1, en los cuatro 
tamaños de empresas micro, pequeñas, medianas y grandes se observa una tendencia decreciente del ROA, lo cual se explica por la alta fluctuación de las utilidades promedio expuesta en la sección anterior. En todas ellas se observa una tendencia decreciente del ROE, debido a la mencionada variabilidad de las utilidades promedio. Se puede apreciar entonces una tendencia de decrecimiento del ROE en relación con el ROA, lo cual significa la presencia de apalancamiento negativo que implica que el endeudamiento perjudica a los propietarios. Las mayores variaciones corresponden a las microempresas comprobando así lo determinado por Solís y Robalino (2019).

Respecto a los cuatro tipos de empresas industriales, comerciales y de servicios, en la Figura 2 se observa una tendencia decreciente del ROA, debido a la considerable fluctuación de las utilidades promedio expuesta en secciones precedentes. En todas las empresas se observa una tendencia decreciente del ROE causada por la elevada oscilación de valores explicada anteriormente.

Por lo expuesto, se evidencia una tendencia de decrecimiento del ROE en relación con el ROA, lo cual implica un apalancamiento negativo, en el cual el endeudamiento no favorece a los propietarios. El mayor impacto negativo se registra en las empresas de servicios. Por el contrario, es en las empresas industriales en donde se presenta un mejor desempeño, panorama similar a lo referido por Coello (2017) y también por Chuncho et al. (2021) en la generalidad de empresas manufactureras ecuatorianas, lo cual converge con lo expuesto por Arévalo et al. (2018) referente a las empresas de construcción especialmente en el año 2017. Por supuesto, entre regiones y provincias existen diferencias debido a diversos factores, tanto internos como externos, y en ese sentido, en el caso de la Sierra central -cantón Riobamba- el desempeño de las empresas de este tipo, aunque ha tenido avances,



Figura 2. Diferencia ROE y ROA - período 2010-2019 - total empresas por tipo. Fuente: Superintendencia de Compañías, Valores y Seguros (2020). 
también han experimentado grandes dificultades según lo evidenciado por Vives y Naranjo (2020).

En base a los resultados obtenidos, se rechaza la hipótesis propuesta, ya que las empresas mayores a 50 años de diverso tamaño y tipo de la provincia de Pichincha que lograron permanecer en el mercado hasta la actualidad no registran un crecimiento sostenido de su activo, patrimonio, utilidades, y por ende de su ROA y ROE, sino que su desempeño es irregular precisamente por factores de dimensión y sector, lo cual se debe no solo a variables endógenas sino también exógenas-macroeconómicas-susceptibles de estudiar en un análisis complementario.

\section{Conclusiones}

Aun cuando las empresas estudiadas son las más longevas y con destacada trayectoria en el Ecuador, en particular en la provincia de Pichincha, lo cual supone el logro de importantes curvas de aprendizaje en términos económicos, legales, de talento humano, de innovación, logísticos, entre otros, se observa que el desempeño económico-financiero, en particular la rentabilidad, ha sufrido una alta variabilidad y momentos de retroceso en los últimos diez años. Esta investigación abre la posibilidad de estudios sobre las causas que han generado los mencionados declives de los índices económicos-financieros.

El conjunto de empresas muestra un incremento de su patrimonio; sin embargo, respecto a sus utilidades se registran grandes fluctuaciones que provocan que tanto el ROA como el ROE de las diferentes organizaciones presenten una tendencia decreciente, especialmente en el año 2012 y el período comprendido entre el 2015 y el 2019, en gran medida debido a la política económica adoptada por el gobierno de turno. Se concluye que, de acuerdo al tamaño de empresas, las más afectadas son las microempresas y en relación al tipo, las más frágiles son las empresas de servicios.

A pesar de que se observa una disminución del nivel de utilidades, y en consecuencia de la rentabilidad en el período estudiado, las empresas longevas realizan esfuerzos por mantenerse en el mercado. En este sentido, el estudio también abre la posibilidad de analizar posteriormente otros aspectos como los factores críticos de éxito que les han permitido a este tipo de empresas permanecer en el mercado hasta la actualidad como ejemplo de fortaleza e innovación.

\section{Fuente de financiamiento de la investigación}

Comisión de Investigación Formativa (CIF) de la Universidad Central del Ecuador.

\section{Contribución de autores}

C.A.O.Z. Idea, revisión de literatura, metodología, análisis de datos, redacción de discusión y conclusiones.

J.A.G.B. Metodología y análisis de datos. 

datos.

L.F.C.B. Metodología y análisis de

\section{Referencias}

Acosta-González, N., y Camelos, E. (2018). Restricciones al crecimiento de la productividad en el Ecuador: Brecha externa y fiscal (2002-2017). Boletín de Coyuntura, (18), 14-17.

Aguiar, I. (2018). Las empresas longevas canarias. Revista Hacienda Canaria de la Universidad de Las Palmas de Gran Canaria, (48), 135-167.

Arévalo, S., Osejo, T., Fonseca, J., y Canchignia, C. (2018). Análisis financiero del sector construcción de las empresas ecuatorianas en el periodo 2016-2017. Revista de investigación SIGMA, 05(2), 39-49. http://dx.doi.org/10.24133/sigma. v5i02.1253

Arregui, G. (1999). Dinero y poder en los origenes de nuestra época. Madrid: Alcal.

Asamblea Nacional del Ecuador. (2018). Reglamento de inversiones del Código Orgánico de la Producción, Comercio e Inversiones. Quito, Ecuador.

Asamblea Nacional del Ecuador. (2019). Código Orgánico de la Producción, Comercio e Inversiones (COPCI). Quito, Ecuador.

Ayala, E. (1993). Resumen de la Historia del Ecuador. Quito: Corporación Editorial Nacional.

Banco Central del Ecuador - BCE. (2020). Estadísticas macroeconómicas. Presentación Coyuntural 2010-2021. Recuperado de https://contenido.bce.fin.ec/documentos/ Estadisticas/SectorReal/Previsiones/IndC oyuntura/IndiceEMcoyuntural.html

Camino, S., Reyes, A., Apraes, D., Bravo, D., y Herrera, D. (2017). Estudios Sectoriales:
MIPYMES y grandes empresas. Superintendencia de Compañías, Valores y Seguros.

Campuzano, J., Cedillo, L., Chuquirima, S., y Romero, W. (2019). Emprendimiento dinámico, crecimiento económico y política pública en el Ecuador. INNOVA Research Journal, 4(3.1), 42-54. https://doi.org/10.33890/innova.v4.n3.1. 2019.1076

Chuncho, L., Uriguen, P., y Apolo, N. (2021). Ecuador: análisis económico del desarrollo del sector agropecuario e industrial en el periodo 2000-2018. Revista Cientifica y Tecnológica UPSE, 8(1), 08-17. https://doi.org/10.26423/ retu.v8i1.547

Coello, D. (2017). ¿Poder de mercado o eficiencia? Determinantes de la rentabilidad del sector manufacturero ecuatoriano durante el periodo post-dolarización. X-pedientes Económicos, 1(1), 56-77.

Comisión Económica para América Latina y el Caribe-CEPAL. (2019). La inversión extranjera directa en América Latina y el Caribe. Santiago: CEPAL.

Crédito y Caución. (2014). Secretos de la longevidad empresarial. Análisis CyC. Madrid, 1 de septiembre.

Fariñas, J.C., y Huergo, E. (2015). Estudios sobre Demografía empresarial en España: tendencias y regularidades. Estudios sobre la economía española, Working Paper 2015/24.

Fierro, L. (2019a). Fortalecimiento de los grupos económicos en el Ecuador en la última década. Revista Economía, 71(114), 35-71. https://doi.org/10.29166/econo mia.v71i114.2222

Fierro, L. (2019b). Los grupos financieros en el Ecuador - 25 años después. Revista PUCE, (108), 139-175. https://doi.org/10. 
26807/revpuce.v0i108.217

Instituto Nacional de Estadística y Censos-INEC. (2017). Panorama laboral y empresarial del Ecuador. Laboratorio de Dinámica Laboral y Empresarial.

Instituto Nacional de Estadística y Censos -INEC. (2020). Boletín Técnico No. 01-2020-IPC, Índice de Precios al Consumidor-Enero 2020. Estadísticas económicas. Recuperado de https:// www.ecuadorencifras.gob.ec

Larrea, C. (2004). Dolarización y desarrollo humano en Ecuador. Íconos, (19), 43-53.

Lascano, G., Méndez, R., y Barcia, R. (2020). El nuevo Código de Comercio ecuatoriano: Reflexiones para el debate. Yachana Revista Cientifica, 9(2), 66-76.

Mejía, S., Pinos, L., Proaño, W., y Rojas, J. (2019). Expectativas empresariales y ciclo económico de la economía ecuatoriana 2007-2018. PODIUM, (36), 1-22. doi:https://doi.org/10.31095/podium.201 9.36.1

Moina-Sánchez, P., Morales-Carrasco, L., y CórdovaPacheco, A. (2020). Crecimiento económico en una región emprendedora en el Ecuador. Retos Revista de Ciencias de Administración y Economía, 10(19), 65-80. https://doi.org/10.17163/ret.n19.2020.04

Parra, J. (2011). Determinantes de la probabilidad de cierre de nuevas empresas en Bogotá. Revista de la Facultad Ciencias Económicas: Investigación y Reflexión, $X I X(1), 27-53$.

Rodríguez, C., y García, M. L. (2020). Culturas organizacionales exitosas. Empresas Mexicanas con más de 50 años de existencia. Universidad Politécnica de Guanajuato.

Sierra, E. (1999). La pirámide en conflicto. Quito: Edidac.
Solís, L., y Robalino, R. (2019). El papel de las PYMES en las sociedades y su problemática empresarial. INNOVA Research Journal, 4(3), 85-93. https://doi.org/10.33890/innova.v4.n3.20 19.949

Superintendencia de Compañías, Valores y Seguros (2020). Portal de información, Sector societario. Recuperado de https://appscvs movil.supercias.gob.ec/PortalInformacio $\mathrm{n} /$ sector_societario.html

Varela, G. (1995). La teoría de la sociedad de Niklas Luhmann. Sociológica, 10(27).

Vives, J., y Naranjo, C. (2020). Análisis económico del cantón Riobamba en Ecuador durante el "Correato". Revista de Ciencias Sociales, (169), 69-90. https://doi.org/ 10.15517/rcs.v0i169.45484

PODIUM No. 40, Diciembre 2021, pp. 89-104

(C) Universidad Espíritu Santo - UEES

ISSN: 1390-5473 e-ISSN: 2588-0969 
